\title{
Un estudio de fase 3 de pirfenidona en pacientes con fibrosis pulmonar idiopática
}

\author{
A phase 3 trial of pirfenidone in patients with \\ idiopathic pulmonary fibrosis
}

Carlos Eduardo Matiz, MD. (1); Alejandro Parra Peña, MD. ${ }^{(2)}$

${ }^{(1)}$ Médico internista, Neumólogo. Profesor asociado Medicina, Universidad El Bosque. Coordinador Comité Enfermedad Pulmonar Intersticial Difusa. Asociación Colombiana de Neumología y Cirugía de Tórax. Director CEEP.

${ }^{(2)}$ Residente de Primer Año de Medicina Interna, Pontificia Universidad Javeriana, Hospital Universitario San Ignacio. Bogotá, Colombia.

Correspondencia: Carlos Eduardo Matiz, correo electrónico: carlosmatiz@hotmail.com. Recibido: 08/08/14. Aceptado: 10/09/14.

\section{Referencia}

King Jr. TE, Bradford WZ, Castro-Bernardini S, Fagan EA, Glaspole I, Glassberg MK et al, for the ASCEND Study Group. N Engl J Med. 2014;370;22.

\section{Pregunta}

¿Es eficaz y segura la terapia con pirfenidona en comparación con placebo en pacientes con fibrosis pulmonar idiopática?

\section{Diseño}

Experimento clínico, doble ciego, multinacional, aleatorizado, controlado con placebo, de fase 3, realizado para comprobar la eficacia y seguridad de pirfenidona en pacientes que padecen de fibrosis pulmonar idiopática.

\section{Periodo de seguimiento}

Semanas planeadas: 52, semanas completadas: 52 .

\section{Lugar}

Realizado en nueve países, 127 centros hospitalarios, distribuidos de la siguiente forma: Estados Unidos (87), Australia (11), Brasil (6), Croacia (2), Israel (5), México (5), Nueva Zelanda (2), Perú (8) y Singapur (1).

\section{Pacientes}

Se incluyeron 555 pacientes con edades entre 40 y 80 años, con diagnóstico confirmado de fibrosis pulmonar idiopática, de al menos seis meses y no más de 48 meses de evolución, con una capacidad vital reducida con un porcentaje entre 50 y $90 \%$ al momento de la selección, una capacidad de difusión mayor al $30 \%$ y menor del $90 \%$, y que fueran capaces de realizar en la caminata de 6 minutos más de $150 \mathrm{~m}$.

Se excluyeron pacientes que tuvieran una explicación conocida para la enfermedad, pacientes con historia de asma y enfermedad pulmonar obstructiva crónica o que estuvieran en lista de espera para trasplante.

\section{Intervención}

Terapia con pirfenidona 2.403 $\mathrm{mg}$ distribuidos en tres dosis iguales con las comidas, e incremento gradual hasta alcanzar la dosis máxima a las dos semanas por un periodo de 52 semanas.

Terapia con placebo durante un periodo de 52 semanas.

\section{Resultados}

En la semana 52, la proporción de pacientes que tuvo una disminución 
de 10 puntos porcentuales o más en el porcentaje del predicho de la capacidad vital forzada (CVF) o que habían muerto, se redujo en un 47,9\% en el grupo de pirfenidona en comparación con el grupo placebo (46 pacientes $[16,5 \%]$ frente a $88[31,8 \%](p<0,001)$.

La proporción de pacientes sin disminución en el porcentaje del predicho de la CVF se incrementó en $132,5 \%$ en el grupo de pirfenidona (63 pacientes $[22,7 \%]$ frente a $27[9,7 \%])$.

La disminución media desde el inicio en la CVF fue de $235 \mathrm{ml}$ en la pirfenidona y $428 \mathrm{ml}$ en el grupo de placebo con diferencia absoluta y relativa de193 y $45,1 \%(p<0,001)$.

La pendiente lineal de descenso de la CVF en la semana 52 fue de $-122 \mathrm{ml}$ con pirfenidona vs. -262 $\mathrm{ml}$ con placebo, con una diferencia absoluta y relativa de $140 \mathrm{ml}$ y $53,5 \%(\mathrm{p}<0,001)$.

Durante el periodo de seguimiento, $25,9 \%$ de los pacientes en el grupo de tratamiento presentaron disminución de 50 metros o más en la caminata de 6 minutos comparado con $35,7 \%$ en el grupo placebo. Se observó reducción relativa del $27,5 \%$ en el grupo de pirfenidona.

En comparación con placebo, pirfenidona redujo el riesgo relativo de muerte o progresión de la enfermedad en un $43 \%$ (cociente de riesgo en el grupo de pirfenidona, 0,$57 ; 95 \%$ intervalo de confianza [IC]: $0,43$ a 0,$77 ; p<0,001)$.

El análisis de todas las causas de mortalidad mostró menos muertes en el grupo pirfenidona que en el placebo, aunque la diferencia no fue significativa. Adcionalmente, 11 pacientes (4,0\%) murieron durante el estudio, en comparación con 20 pacientes $(7,2 \%)$ en el grupo placebo (HR 0.55; 95\% IC, 0 en el grupo de pirfenidona $26-1,15 ; \mathrm{p}=0,10)$.

Se produjeron muertes por fibrosis pulmonar idiopática en 3 pacientes $(1,1 \%)$ y 7 pacientes $(2,5 \%)$ en los grupos de pirfenidona y placebo, respectivamente $(\operatorname{HR} 0,44$, IC 95\%, 0,11-1,72, p=0,23).

\section{Conclusión}

En el estudio ASCEND (Assessment of Pirfenidone to Confirm Efficacy and Safety in Idiopathic
Pulmonary Fibrosis), diseñado con el fin de confirmar la seguridad y eficacia de la pirfenidona en el tratamiento de pacientes con fibrosis pulmonar idiopática, se demostró que los pacientes tratados con pirfenidona en comparación con el grupo placebo, presentaban menor disminución de la CVF, que es un fuerte predictor de alto riesgo de mortalidad en pacientes que padecen esta enfermedad (1).

Así mismo, en pacientes con fibrosis pulmonar idiopática la pirfenidona redujo la proporción de quienes presentaban un descenso de $50 \mathrm{~m}$ o más en la prueba de caminata de 6 minutos, que es un predictor independiente de mortalidad en este tipo de pacientes.

De acuerdo con el estudio se puede concluir que la pirfenidona es un medicamento eficaz y seguro para el tratamiento de pacientes con fibrosis pulmonar idiopática, reflejado en las pruebas de función pulmonar, mejoría de la tolerancia al ejercicio y efectos secundarios leves y tolerables.

\section{Comentario}

La fibrosis pulmonar idiopática es una enfermedad progresiva, crónica y fatal caracterizada por la pérdida irreversible de la función pulmonar, que aunque tiene periodos de estabilidad, su progreso continuo es inevitable. Su prevalencia ha aumentado en los últimos años, con una supervivencia de tres a cinco años desde el diagnóstico, muy similar a patologías como el cáncer.

Se considera que la fisiopatología de la fibrosis pulmonar idiopática se caracteriza por un depósito progresivo de colágeno y de otras moléculas de la matriz extracelular. Antes se pensaba que se debía a una respuesta inflamatoria constante que llevaba a fibrosis pulmonar crónica posterior; sin embargo, la inflamación, ya no se considera un proceso crucial puesto que las terapias antiinflamatorias han proporcionado pocos beneficios para los pacientes.

Se ha hecho evidente que el evento crucial es el comportamiento anormal de las células epiteliales alveolares, que secundario a una lesión repetitiva de las mismas, provocan su activación, haciendo que se recluten células inmunes y fibroblastos dentro 
del microambiente pulmonar. El comportamiento aberrante de las células epiteliales alveolares, junto con la migración de células inmunes y fibroblastos, generan la actividad latente de TGF-B1 (del inglés transforming growth factor beta-1), como también otros factores profibróticos, los cuales promueven la diferenciación de fibroblastos y células epiteliales alveolares en miofibroblastos, que conllevan sobreproducción de células de matriz extracelular en el pulmón.

Hasta la aprobación de pirfenidona en 2008, ningún fármaco ha demostrado ser eficaz para el tratamiento de la fibrosis pulmonar idiopática, aunque se han realizado varios estudios clínicos de agentes potenciales. Nuevos tratamientos para la fibrosis pulmonar idiopática, entre los que se incluyen bosentan (antagonista de endotelina), imatinib (inhibidor de la tirosin kinasa), sildenafil (inhibidor de fosfodiesterasa), etanercept (anticuerpos contra el FNT- $\alpha$ ) e interferón INF $\Upsilon-1 b$ han sido evaluados en ensayos clínicos aleatorizados, pero ninguno de estos ha demostrado una significancia estadística en el tratamiento. Además, un reciente estudio aleatorizado (PANTHER - IPF) con prednisona, azatioprina y $\mathrm{N}$-acetilcisteína demostró que la triple terapia no era beneficiosa y que por el contrario aumentaba el riesgo de muerte y hospitalizaciones en comparación con placebo.

La pirfenidona es un nuevo fármaco en presentación oral con propiedades antifibróticas, antiinflamatorias y antioxidantes. Se ha demostrado que puede modular la expresión de factores profibróticos y citoquinas proinflamatorias y que suprime potencialmente la producción de especies reactivas de oxígeno (2).

$\mathrm{Su}$ mecanismo de acción se basa en que inhibe el factor de necrosis tumoral $\alpha$ (FNT- $\alpha)$ y su regulación postranscripcional y adicionalmente mejora la producción de IL 10 contribuyendo en su actividad antifibrótica, la cual está potenciada ya que es un potente inhibidor del TGF B, que es una de las citoquinas profibróticas más estudiadas. En el pulmón es producida por una amplia variedad de tipos celulares como macrófago alveolar, células epiteliales alveolares activadas, neutrófilos, células endoteliales, fibroblastos y miofibroblastos. El TGF B induce la proliferación de macrófagos y fibroblastos por la expresión del factor de crecimiento derivado de plaquetas (PDGF), que a su vez presenta inhibición directa por la pirfenidona (3).

La eficacia clínica y seguridad de este medicamento han sido demostradas en cuatro estudios aleatorizados, doble ciego, placebo controlado, incluyendo uno en fase 2 y uno en fase 3, llevados a cabo en Japón y dos multinacionales en fase 3, realizados en Norte América, Europa y Australia (CAPACITY) (4).

En un meta-análisis independiente de datos de los tres estudios de fase 3, Spagnolo et al., reportaron una mejoría en el tiempo de sobrevida libre de progresión de la enfermedad en pacientes tratados con pirfenidona, y su administración fue segura y generalmente bien tolerada. En el estudio CAPACITY, los efectos adversos gastrointestinales y en piel, fueron los más representativos, sin embargo, fueron de leves a moderados y no impidieron la continuación del estudio.

En el estudio ASCEND se confirmó la eficacia y se observó un perfil de seguridad favorable; además, en general, fue bien tolerado en pacientes con fibrosis pulmonar idiopática.

\section{Conflictos de intereses}

Los autores declaran no tener conflictos de interés.

\section{Bibliografía}

1. Talmadge EK. A phase 3 trial of pirfenidone in patients with idiopathic pulmonary fibrosis. N Engl J Med. 2014;370;22.

2. Takeda Y. Efficacy and safety of pirfenidone for idiopathic pulmonary fibrosis. Patient Preference and Adherence. 2014;8:361-70.

3. Talmadge EK. Comprehensive assessment of the long-term safety of pirfenidone in patients with idiopathic pulmonary fibrosis. Respirology. 2014;19:740-7.

4. Cano E. Fibrosis pulmonar idiopática: tratamiento con pirfenidona. Arch Bronconeumol. 2012;48(Supl 2):16-8. 